\title{
A novel approach to control the jointed arm robot by tracking the position of the moving object on a conveyor using an integrated computer vision system
}

\author{
Pramod Kumar Thotapalli*, CH. R. Vikram Kumar** and B. ChandraMohana Reddy*** \\ * R esearch Scholar, JNTUA Research Centre, NBKRIST, Vidyanagar, Nellore, AP, India \\ ** Professor, Department of Mechanical, NBKRIST, Vidyanagar, Nellore, AP, India \\ *** Associate Professor, Department of Mechanical, JNTUACE, Anantapuramu, AP, India \\ **Corresponding Author: cvikramkumar@gmail.com
}

Submitted :09/05/2020

Revised :24/01/2021

Accepted : 16/03/2021

\begin{abstract}
Computer vision algorithms play a vital role in developing self-sustained autonomous systems. The objective of the present work is to integrate the robotic system with a moving conveyor using a single camera by adopting a Gaussian Mixture Model (GMM) based background subtraction method. In this work, a simple web camera is placed above the work cell to capture the continuous images of the moving objects on the conveyor along with a jointed arm robot connected to a microcontroller through the computer. The position of the object with time and its features are extracted from the captured image frames by subtracting its background using the Gaussian Mixture Model (GMM). The output images of GMM are further processed by image processing techniques to extract the features like shape, color, and center coordinates. The extracted coordinates of objects of interest are used as input parameters to the controller to activate the base rotation of a joint arm robot to perform different manipulations. The developed algorithm is evaluated on an indigenously fabricated work cell integrated with a computer vision setup.
\end{abstract}

Keywords: Computer vision; Control; Conveyor; Robot; GMM.

\section{INTRODUCTION}

Computer vision helps the robots perform the task independently in any environment. In industrial applications, vision-assisted systems can improve productivity with consistent quality in the product and processes. Vision-based artificial intelligence can assist in many applications like the inspection, planning, and controlling the robot. It enhances the degree of automation of the production system. In manufacturing, moving object manipulations are needed for orientation, positioning to perform assembly, sorting, and many other operations involved in handling the product from one workstation to another. The extracted features help the robot grasp the object of interest and also perform the various manipulations as per the requirements. In this context, a novel approach is proposed to extract the features of moving objects using GMM based background subtraction to develop an integrated vision for tracking and controlling the robot. 
Tracking with the help of the vision system plays an important role in segmenting the features of the moving objects from its background cluster. An effective tracking vision algorithm is necessary to exclude a product from its background in developing many industrial robotic applications. Background subtraction is one of the suitable techniques to extract the objects from the background. Various background subtraction techniques have been developed for real-time tracking and detection applications. They primarily focused on traffic monitoring, surveillance, and human-machine interaction (S Cheung Sen-Ching and Chandrika Kamath, 2004). The visual tracking results help operate and control the joint arm robot to perform various operations like pick and place, inspection, false identification, orientation, and positioning within the robot's work volume. Abbood et al. (2020) developed an interactive vision-based robot for real-time object sorting. In this approach, color and shape segmentation algorithms are used, and the range of color threshold filter for individual color is used, but this algorithm fails to extract the other objects with multiple colors. The major drawback in this approach is that the change in the illumination affects the accuracy of the object coordinates. Chen et al. (2019) developed comprehensive vision system for dynamic measurement, including four modules (operator face authentication, gesture remote control, abnormal entry detection, and moving target tracking that makes the manufacturing intelligent safe, efficient, and safe using artificial intelligence applications as intelligent robot arm). A slight variation in the illumination leads to a false result in the temporal difference algorithm in detecting the abnormal entry. Thotapalli et al. (2020) developed a feature extraction methodology for moving objects based on background subtraction to handle multiple parameters like illuminations effect, objects with the same background color.

To track the object's movement, several researchers developed different techniques for tracking the object. They are generally categorized into basic, parametric, and nonparametric methods. The basic method mainly focused on the temporal difference between the previous and current frames pixel's intensities (Christof Ridder et al., 1995). However, it fails to track while moving slowly or stopping the object for a certain amount of time. To overcome this problem, it is necessary to define a time between the consecutive frames. It also fails to work during the distinctive motion characteristics, namely, high speed. Because of weak object localization and overlap (Chris Stauffer and W Eric L Grimson., 1999), parametric and nonparametric methods are suggested to model the complex backgrounds for different scenarios

In the parametric method, each pixel is modeled with mean and variance to model the background. It may be a model with single or multiple Gaussians. In a single Gaussian approach, each pixel's probability density functions (PDF) in a current frame are associated with the reference value to discriminate the background from the foreground (P Wayne Power and Johann A Schoonees 2002). GMM is a parametric model with multiple Gaussians. It prevents the foreground data from the background by assigning each pixel with the associated weights to effectively update the background (Alan M McIvor., 2000). Nonparametric methods were developed along with the parametric methods. These methods are a discrete estimation of the probability distribution to define the image background model (Thierry Bouwmans, Fida El Baf, Bertrand Vachon., 2008). It is done based on Kernel Density Estimation (KDE) to estimate the PDF associated with the foreground and background. It fails to model periodic movements of the object for both long and short-term events because it cannot hold a large amount of data to update the model (P. St-Charles et al., 2015). Massimo Piccardi (2004) reviewed multimodal distribution for parametric (Mixture of Gaussians) and nonparametric (Kernel Density Estimation) methods based on computational speed, memory, and detection accuracy. Both approaches can model the probability density functions of background in general cases. However, KDF requires a high memory. GMM has become quite popular in modeling complex backgrounds due to improvements in updating the model and distance criteria to discriminate against the foreground-background.

The proposed method in this paper is intended to extract multiple feature attributes like size, shape, orientation, texture, color, pattern, and location of the objects moving on the conveyor and controlling the robot for sorting the objects or detecting the defective objects based on attributes using the single overhead vision system. In this work, an attempt was made to apply a GMM based background subtraction technique to develop an integrated vision system 
to control the jointed arm robot based on the object tracked position using overhead vision system (web camera) connected through the computer. The position of the end effectors is defined with reference to the object center coordinates identified in a vision system by applying the inverse kinematics to compute the remaining joint angles of a robot for pick and place operation. The program for the algorithm is developed using MATLAB software.

\section{METHODOLOGY}

The schematic layout of the work cell is shown in Fig. 1. The layout illustrates the view field of the work cell, which consists of a jointed arm robot, conveyor belt, and an overhead stationary vision system (web camera). All are connected through the microcontroller (Arduino UNO R3) to form an integrated computer system. Appropriate placement of the vision system is most important to tack the objects in the work volume. It is placed above the work cell at height, such a way to capture the entire working volume of a robot. The computer triggers the vision system (web camera) to capture the real-time images of the workspace. These images are stored and further processed using region properties to extract center coordinates. Let the field view of the stationary web camera be shown as a rectangular box with $\mathrm{X} \& \mathrm{Y}$ coordinates of the image size varying from $(0,0)$ to $\left(\mathrm{X}_{\max }, \mathrm{Y}_{\max }\right)$, and an image frame size of $640 \mathrm{X} 480$ in terms of pixels is used in the present algorithm. The belt conveyor and bins are placed within the maximum reach of the robot end effectors. Let $\mathrm{A}\left(\mathrm{X}_{\mathrm{b}}, \mathrm{Y}_{\mathrm{b}}\right)$ be the fixed base center of the robot, $\mathrm{B}$ $\left(\mathrm{X}_{\mathrm{i}}, \mathrm{Y}_{\mathrm{i}}\right)$ be the center coordinates of the identified object 'B' moving over the belt conveyor, $\mathrm{C}\left(\mathrm{X}_{\mathrm{p}}, \mathrm{Y}_{\mathrm{p}}\right)$ be the initial home position of the robot end-effector, and $\mathrm{D}\left(\mathrm{X}_{\mathrm{t}}, \mathrm{Y}_{\mathrm{t}}\right)$ be the location of the target bin. As represented in the layout in Fig. 1, the conveyor belt is moving along the $\mathrm{X}$-axis of the image frame, and objects $\mathrm{B}\left(\mathrm{X}_{\mathrm{i}}, \mathrm{Y}_{\mathrm{i}}\right)$ are also moving over the conveyor along the $\mathrm{X}$-axis of the image frame. Once it is entered into the image field, the ' $\mathrm{X}^{\prime}$ ' coordinate of the object varies from 'Zero' to 'Maximum,' and ' $Y_{i}$ ' coordinate is constant. The algorithm continuously extracts the coordinates moving object $\mathrm{B}\left(\mathrm{X}_{\mathrm{i}}, \mathrm{Y}_{\mathrm{i}}\right)$ over the conveyor. The work limits of the robot over the conveyor are shown in Fig. 1. In the case of any vibrations, slipping or drifting of belt conveyor may alter the position of the object $\left(\mathrm{X}_{\mathrm{i}}\right.$, $\mathrm{Y}_{\mathrm{i}}$ ). Normally, the conveyor is fitted with an encoder to track the position of the component. The consecutive coordinates of the tacked object help analyze the condition of the conveyor. For a vibration-free conveyor system, the $Y_{i}$ coordinate of the object is constant while entering and leaving the view filed. Nevertheless, the vision algorithm can extract the coordinates from the image frame.

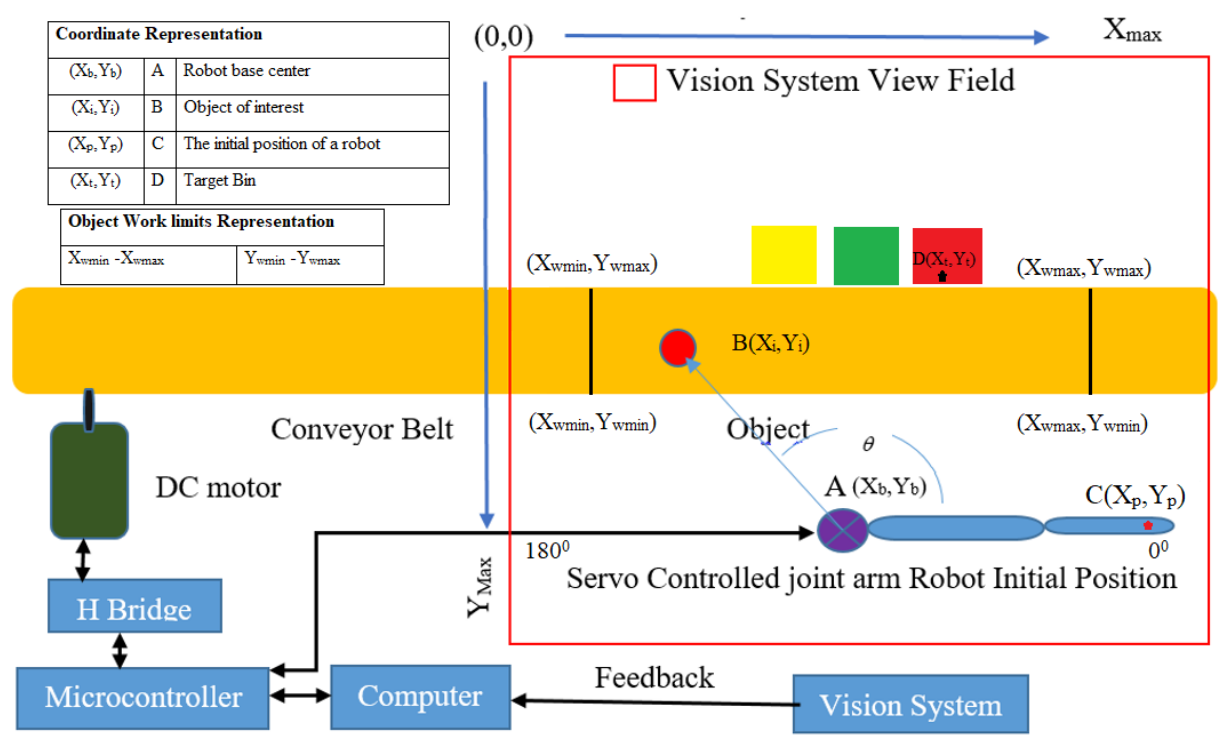

Figure 1. Schematic layout of the robotics work cell. 


\section{Definition of the Robot Home Position in the Work Volume}

In this work, a prototype robot was used to interface with the moving conveyor for pick and place application through a vision system. To actuate the robot from the initial position to pick the object, it is necessary to define the initial reference position of the robot before starting the task. The initial position of the robot is designed using an image frame captured by an overhead camera, as shown in Fig. 2. To define a reference point on the robot, first mark a reference point ' $\mathrm{A}$ ' at the center of the robot base and consider a point ' $\mathrm{C}$ ' on the robot arm. Using image processing, the coordinates of the point are extracted as $A\left(X_{b}, Y_{b}\right)$ and $C\left(X_{p}, Y_{p}\right)$. These two coordinates were extracted from the captured image frame using the pixel info tool at the beginning of the cycle for a particular application or before the algorithm's initialization. The points ' $\mathrm{A}$ ' and ' $\mathrm{C}$ ' form a Vector $\overrightarrow{A C}$ represent the robot's initial position. These two points are fixed for an image frame of a particular pixel size when it captures from the stationary overhead vision system at a constant position.

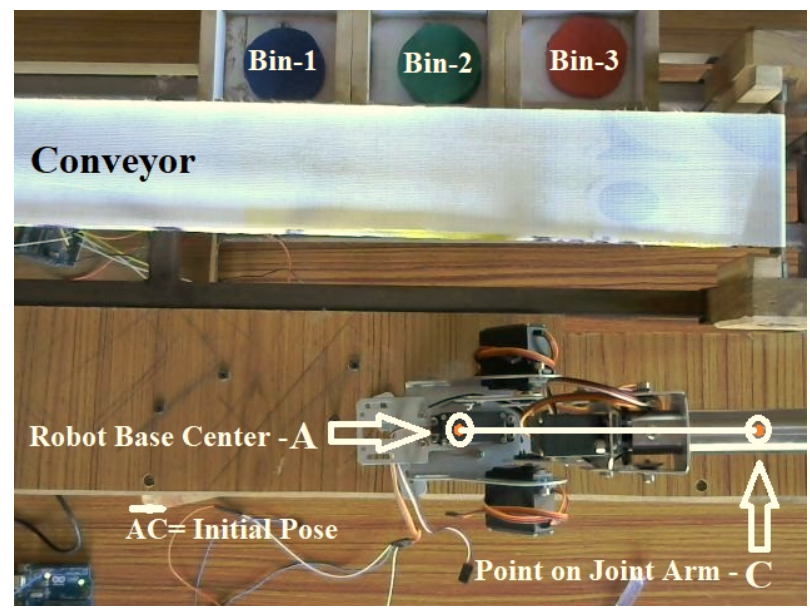

Figure 2. Extraction of Initial Position Coordinates of the Robot from the Image Frame.

\section{Initialize the GMM for background subtraction}

GMM is one of the parametric methods based on pixel attributes to model multimodel backgrounds. Each pixel attributes a value represented as a Gaussian component density. It is characterized as a mixture of K Gaussian component densities. It is taken as three in the present work (Chris Stauffer and W Eric L Grimson., 1999). Pixels are persistence in terms of mean value, and variance establishes a match among the current image frames to reference image frame at a time. The match of respective pixels is defined as the Mahalanobis distance threshold (K. Goyal and J. Singhai, 2017). It is an input parameter to govern the sensitivity to differentiate between the foreground and background. The illumination variations can handle in these situations by updating the background with current mean, high variance, and low prior weight of respective pixels (Chris Stauffer and W Eric L Grimson., 1999). The following methodology represents the subtraction of background from foreground

Let ' $\mathrm{X}$ ' be the image frame and each pixel in the image represented as $\left(x_{1}, \ldots \ldots \ldots, x_{n}\right)$. In this method, a mixture of K Gaussian distributions is used to models each pixel color. The PDF of the kth Gaussian of the pixel at time $\mathrm{t}$ is given as $P\left(X_{t}\right)$ from the frame at the time. The probability of occurrence of a pixel $\mathrm{x}$ at $\mathrm{t}$ is represented by 


$$
P\left(X_{t}\right)=\sum_{k=1}^{K} W_{k, t} * \eta\left(X_{t}, \mu_{k, t}, \Sigma_{k, t}\right)
$$

where $P\left(X_{t}\right)$ is the PDF of $X_{t}$ in a parametric form and composed of a mixture of Gaussians of each pixel at time t.

$\mathrm{K}$ is the number of Gaussian distributions, $W_{k, t}$ is the estimate of the weight of the $\mathrm{k}^{\text {th }}$ Gaussian at time $\mathrm{t}$, $\mu_{k, t}$ is the mean value of the $\mathrm{k}^{\text {th }}$ Gaussian at time $\mathrm{t}$, and $\Sigma_{k, t}$ is the covariance matrix of the $\mathrm{k}^{\text {th }}$ Gaussian probability density function.

$$
\begin{array}{r}
\eta\left(X_{t}, \mu_{k, t}, \Sigma_{k, t}\right) \text { is the } \mathrm{k}^{\text {th }} \text { Gaussian PDF is represented as } \\
\eta\left(X_{t}, \mu_{k, t}, \Sigma_{k, t}\right)=\frac{1}{(2 \pi)^{\frac{1}{2}}\left|\Sigma_{k, t}\right|^{\frac{1}{2}}} e^{-\frac{1}{2}\left(X_{t}-\mu_{t}\right)^{T} \Sigma_{k, t}^{-1}\left(X_{t}-\mu_{t}\right)}
\end{array}
$$

For computational reasons, it assumed that the color components are independent and have the same variances. Therefore, the covariance matrix is of the represented Andrews Sobral and Antoine Vacavant (2014).

$$
\Sigma_{k, t}=\sigma_{k, t}^{2} I
$$

To consider the pixel as a background or foreground, the threshold distance in the form of Mahalanobis (Roy De et al. 2000).

$$
\text { Mahalanobis }=\sqrt{\left(\left|X_{t}-\mu_{t}\right|^{T}\right)\left(\Sigma_{t}^{-1}\right)\left(\left|X_{t}-\mu_{t}\right|\right)}
$$

To interpret the illumination variations in the scene, the covariance and mean of each pixel are iteratively updated as follows (Chris Stauffer and W Eric L Grimson, 1999).

$$
\begin{aligned}
& \Sigma_{t+1}=(1-\alpha) \cdot \Sigma_{t}+\alpha\left(X_{t}-\mu_{t}\right)\left(X_{t}-\mu_{t}\right)^{T} \\
& \mu_{t+1}=(1-\alpha) \mu_{t}+\alpha X_{t}
\end{aligned}
$$

If a new pixel $\mathrm{x}$ at time $\mathrm{t}$ is checked against the existing $\mathrm{K}$ Gaussian distributions of reference pixel Mahalanobis less than the threshold distance, then it is defined as a match and considered as background. If none of the K Gaussian distributions match the current pixel value, then the least probable distribution is replaced by mean, high variance, and low prior weight updated in the background image (Chris Stauffer and W Eric L Grimson, 1999).

$$
W_{k, t}=(1-\alpha) W_{k, t}+\alpha \cdot M_{k, t}
$$


where $\alpha$ is a learning rate to update the background of the image frame, and $M_{k, t}$ is 1 for the match; otherwise, $M_{k, t}$ is 0 . Background $\left(\mathrm{B}_{1}\right)$ Gaussian distribution exceeds a certain threshold and is considered background distribution (Ridder et al., 1995).

$$
B_{1}=\arg \min _{b}\left(\sum_{k=1}^{b} W_{k}>T_{h}\right)
$$

where $T_{h}$ is the input parameter assigned as threshold distance.

\section{Activation of the Robot Base}

The coordinates of the moving object $\mathrm{B}(\mathrm{Xi}, \mathrm{Yi})$ are extracted using region properties as shown in Fig. 2. If the coordinates of the object satisfy the conditions $\mathrm{Xwmin}<\mathrm{Xi}<\mathrm{Xwmax}$ and $\mathrm{Ymin}<\mathrm{Yi}<\mathrm{Y} w \max$, which indicates that the object lies within the work volume of the robot; otherwise, the object is lying outside the reach of the robot over the conveyor. Once the object enters onto the conveyor within workspace of the robot, the controller actuates the robot to pick the object. The location of the object with time over the conveyor is determined by extracting consecutive object coordinates at different time intervals on the conveyor. These coordinates are sent through the microcontroller to actuate the robot to pick the object. The object image frame's extracted coordinates, i.e., B ( $\mathrm{X}_{\mathrm{i}}$, $\left.\mathrm{Y}_{\mathrm{i}}\right)$, and the robot base center are $\mathrm{A}\left(\mathrm{X}_{\mathrm{b}}, \mathrm{Y}_{\mathrm{b}}\right)$. The vector $\overrightarrow{A B}$ represents the final position of the robot for picking operation. As already mentioned, the vector $\overrightarrow{A C}$ represents the home position, as shown in Fig. 2. Both vectors are used to calculate the angle between the final and home position in the image frame. Then, the computer triggers the controller to actuate the robot base based on the computed angle between them. By using planer inverse kinematics, the remaining joint angles of the robot are determined to reach the end effectors to the target position. With an integrated vision system, the 3D work volume of the robot is controlled by converting it into a $2 \mathrm{D}$ work plane to control the robot's base rotation.

\section{RESULTS AND DISCUSSIONS}

In this work, GMM based background subtraction algorithm is developed to interface with a moving conveyor to pick the objects based on interest. To evaluate the algorithm, a simple conveyor system was fabricated and integrated with a prototype robot and vision system. In this work, the objects are tracked on the conveyor using the GMM subtraction technique. The features of the moving object are extracted by subtracting its backgrounds. To subtract image from the background, the initial reference frame is captured using a vision system without any object moving on the conveyor, as shown in Fig. 4(a). Each pixel in the reference image frame checked against the consecutive images with time depends on the conveyor's speed. The current image frame consisting of the objects is shown in Fig. 4(b). The background subtraction process is done by computing and comparing the respective mean and covariance of particular pixels information in both reference and current frames. Subtracting each pixel of the current frame by reference extracted the newly appeared objects, as shown in Fig. 4(c). The subtracted image is further processed with imaging techniques like color thresholding, region properties, boundary segmentation, and pattern extraction to extract particular interest information. The performance of the algorithm compared with other background subtraction techniques Gaussian is superior in distinguishing the features of the object even in slightly illumination changes and also able to separate the object having a color similar to the background. The computational speed of the algorithm depends on the size of the image frame (Thotapalli et al., 2020). 


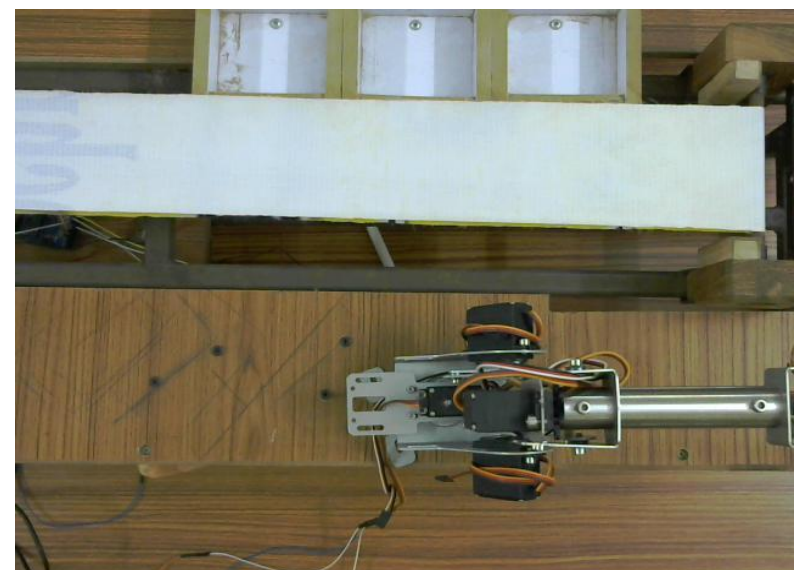

(a) Reference Image without objects

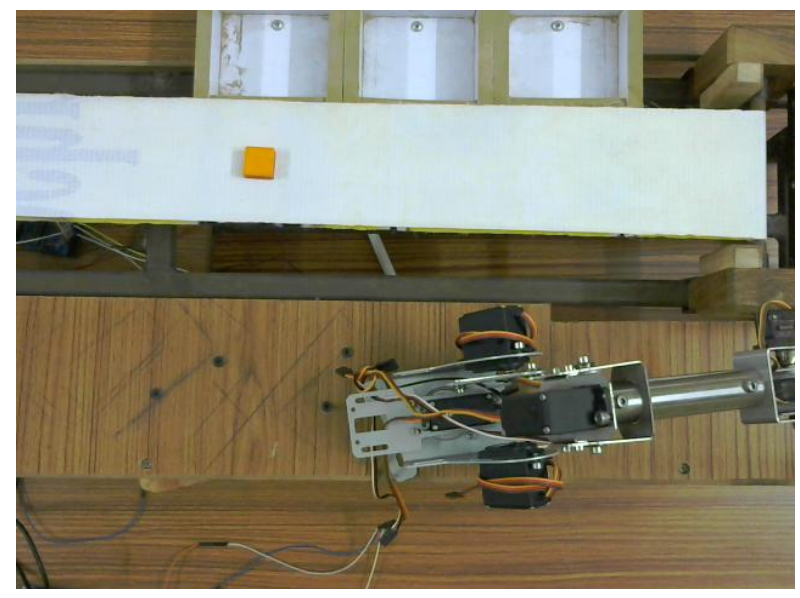

(b) Current image frame with objects

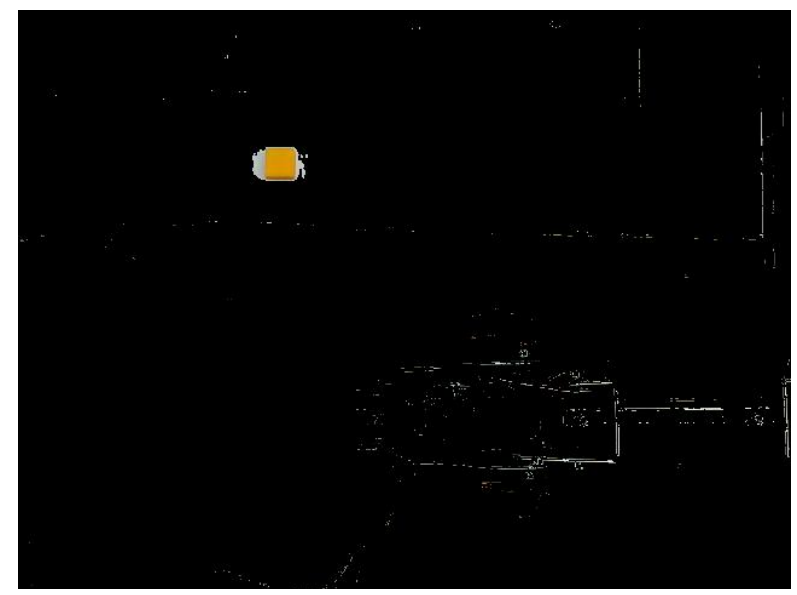

(c) Subtracted image frame

Figure 4. Feature Extraction of Moving object based on Background subtraction. 
The objects feature extraction is extremely useful in developing applications based on segmentation techniques like color, shape, coordinates, patterns, and size. Example color segmentation is used to extract objects based on color for sorting operation. The position of the robot base (A) and a reference point on the robot arm (C) and the placing position of the object or target bin (D) is constant, and the coordinates of these points are determined using image frame in terms of pixels before executing the algorithm and are indicated in Table. 1. Let the object be randomly moved over the conveyor, and the location of the center coordinates of the object at any time instant is determined as $\mathrm{B}\left(\mathrm{X}_{\mathrm{i}}, \mathrm{Y}_{\mathrm{i}}\right)$. The rotation of the base required at particular time instant is calculated by considering the vectors $\mathrm{AC}$ and $\mathrm{AB}$ as shown in Fig. 5.

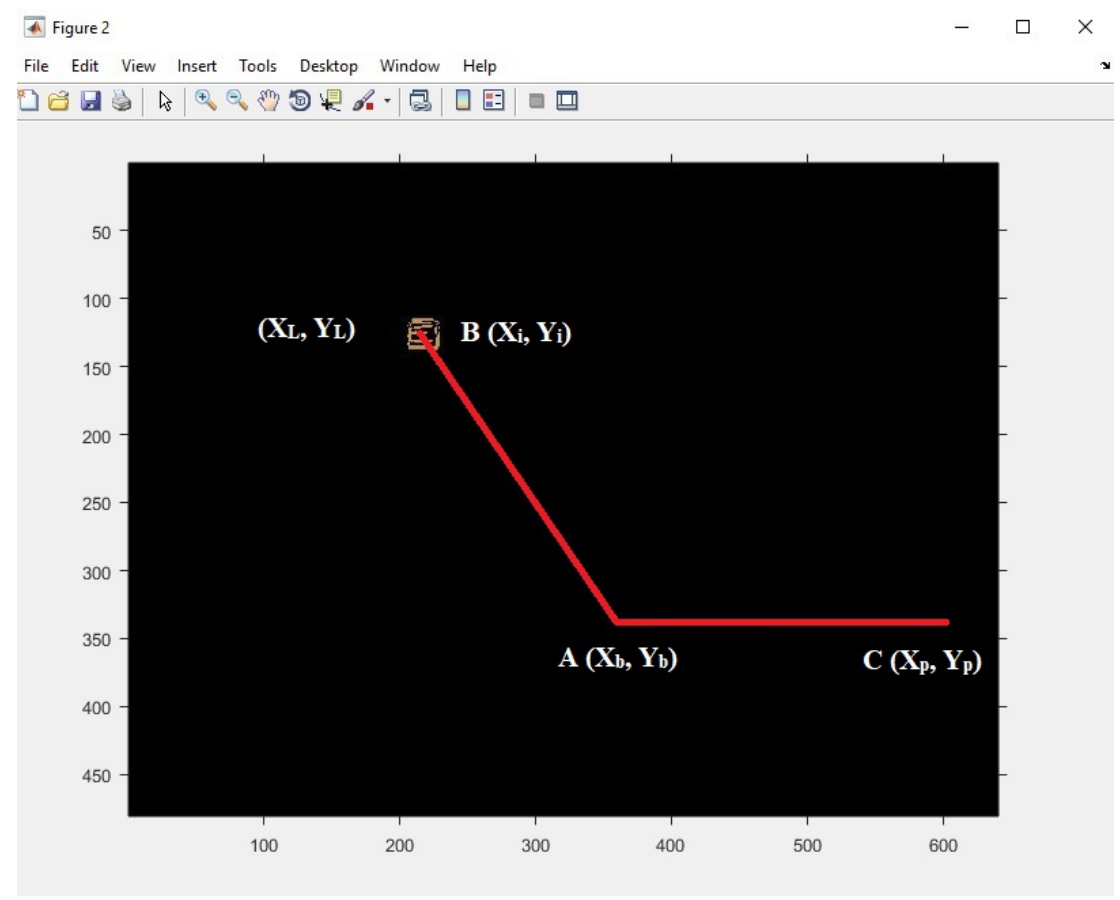

Figure 5. Extracted object coordinates for Robot tracking.

The pixel distance between the extracted points is calculated as shown in Table 2. The extracted distance from the image indicates the pixel distance. Calibration is required to covert these pixel distances in terms of real distance to determine the conveyor speed and to actuate the robot. The calibration is carried out by keeping the camera at a constant height of $700 \mathrm{~mm}$ from the conveyor. The conversion factor to convert the real coordinates with the image coordinates is obtained as 1.36.

Table 1. Fixed coordinates of the image frame.

\begin{tabular}{|c|c|c|c|}
\hline & & $\mathrm{X}$ in pixels & $\mathrm{Y}$ in pixels \\
\hline Base Center of Robot & $\mathrm{A}\left(\mathrm{X}_{\mathrm{b}}, \mathrm{Y}_{\mathrm{b}}\right)$ & 356 & 336 \\
\hline A point on Joint Arm of Robot & $\mathrm{C}(\mathrm{Xp}, \mathrm{Yp})$ & 600 & 336 \\
\hline Target Bin & $\mathrm{D}(\mathrm{Xt}, \mathrm{Yt})$ & 500 & 90 \\
\hline
\end{tabular}


Table 2. Linear distance between the points.

\begin{tabular}{|c|c|c|c|}
\hline Distance between the points & AB & AC & AD \\
\hline Pixels & 215.32 & 244 & 285.04 \\
\hline Real distance (mm) & 158.32 & 179.41 & 209.58 \\
\hline
\end{tabular}

In order to pick the object over the conveyor, it is necessary to determine the position of the objects with the respective time, and it also helps determine the speed of the conveyor. The conveyor speed is calculated based on the object's consecutive position with time. The schematic representation of the positions of the object at different time intervals is shown in Fig. 6. Let the object be located at position 1 (P1) any time instant, object from P1 to the position $(\mathrm{P} 2)$, position (P3), and position (P4) in time intervals time $t_{1}$ and $t_{2}$ as shown in Fig. 6 . In each position, the object coordinates are extracted using image subtraction as given in Table 3 . The time required to process the image can be minimized by reducing the pixel size of the image. If the size frame is reduced from 640X480 to $320 \mathrm{X} 240$, the accuracy of the extracted center coordinates of the object will be also reduced. Based on the variation in the object's location with time, the conveyor speed is determined as shown in Table 3.

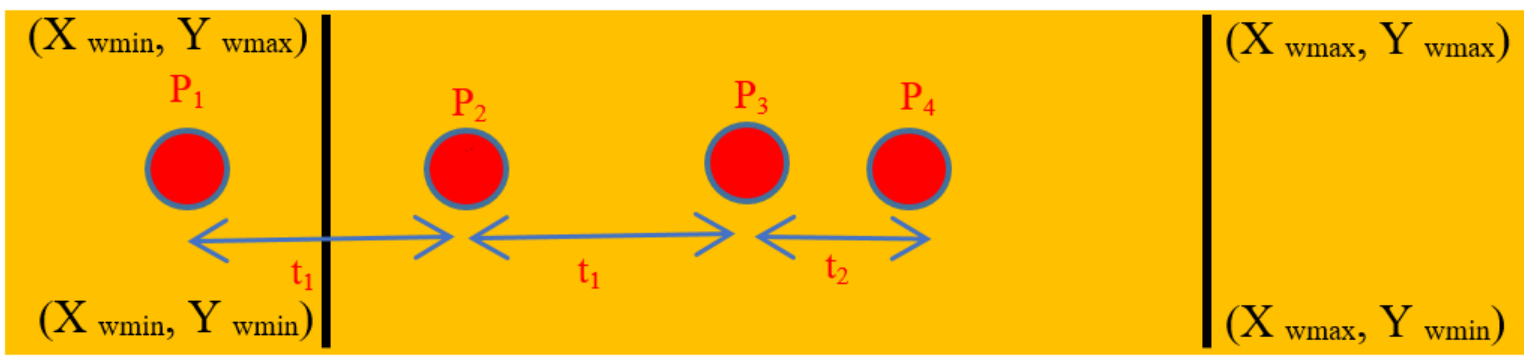

Figure 6. Schematic representing the change in the position of the object on the conveyor with time.

Table 3. Point of intersection for robot pick-up while moving.

\begin{tabular}{|c|c|c|c|c|c|}
\hline \multirow{2}{*}{\multicolumn{2}{|c|}{ Image Size }} & \multicolumn{2}{|c|}{$640 \times 480$} & \multicolumn{2}{|c|}{$320 \times 240$} \\
\hline & & $\mathrm{X}$ & Y & $\mathrm{X}$ & Y \\
\hline $1^{\text {st }}$ position of the object $\left(\mathrm{P}_{1}\right)$ & \multirow{4}{*}{ 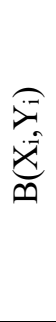 } & 89.5 & 119.2 & 90.03 & 62.6 \\
\hline $2^{\text {nd }}$ Position of the Object $\left(\mathrm{P}_{2}\right)$ & & 225 & 120 & 125.1 & 63 \\
\hline $3^{\text {rd }}$ location object coordinates $\left(\mathrm{P}_{3}\right)$ & & 360.2 & 121.01 & 160.17 & 62.03 \\
\hline $\begin{array}{l}\text { Object pickup point or robot intersection point with the } \\
\text { object }\left(\mathrm{P}_{4}\right)\end{array}$ & & 368.05 & 121.01 & 165.32 & 62.03 \\
\hline Computed angle for robot pick up the object & & \multicolumn{2}{|c|}{86.79} & \multicolumn{2}{|c|}{99.56} \\
\hline Speed of conveyor $(\mathrm{mm} / \mathrm{sec})$ & & \multicolumn{2}{|c|}{16.99} & \multicolumn{2}{|c|}{16.95} \\
\hline
\end{tabular}


The point of intersection is a point on the conveyor from which the robot needs to pick the object calculated based on the conveyor speed and capability of the robot; at the particular instance, the point of intersection is calculated as represented in Table 3. Intersection coordinates are 7.85 pixels in the x-direction. After determining the intersection point, the robot shoulder and elbow's required joint angle are calculated using inverse kinematics as represented in Table 4.

Table 4. Inverse Kinematics for 2R for object picking for 640X480.

\begin{tabular}{|c|c|}
\hline $1^{\text {st }}$ Link Length $(\mathrm{m})$ & 0.12 \\
\hline $2^{\text {nd }}$ Link Length $(\mathrm{m})$ & 0.14 \\
\hline Object distance (m) & 0.158 \\
\hline Theta 1 (degree) & 41.70 \\
\hline Theta 2 (degree) & 32.01 \\
\hline
\end{tabular}

The major advantages of the image subtraction technique are that it will overcome the variations in the object location due to inaccuracies in the conveyor. The accuracy of the object location is very low if the deviation of the conveyor is high. The object of size $20 \mathrm{~mm}$ X $20 \mathrm{~mm}$ X 5mm is used in this work. The accuracy, precision, and speed of the algorithm are crucial for industrial applications. The accuracy in picking the object depends on the type of robot used. The accuracy of object grasping mainly depends on the resolution of the image. High resolution requires high computational time, but the success rate is high compared with the low resolution. It is observed that the computational time increased with the increase in resolution of the image and the application, as shown in Table 6. A low-resolution image results in a loss of bit information of the object's pixel and leads to error in extracting the center coordinates due to sharpness and low threshold.

Table 6. Computational Speed and Accuracy Calculation for positioning to pick the object at different resolutions.

\begin{tabular}{|c|c|c|c|}
\hline & High & Medium & Low \\
\hline Size of the image & $1280 \times 720$ & 640 X480 & $320 \times 240$ \\
\hline Computational Time (GMM) (seconds) & 28.68 & 5.8629 & 3.0423 \\
\hline No of trails & 5 & 5 & 5 \\
\hline No of successful & 5 & 3 & 2 \\
\hline Failure & 0 & 1 & 3 \\
\hline
\end{tabular}

The new methodology was developed to extract objects' features under dynamic conditions like moving and slight variations of illumination in indoor conditions. The vision-assisted integrated systems proposed in this work help the robot controller detect various target points and separate different featured components. It allows a robot to 
perform multiple manipulations for different applications. The approach can be extended by adding postprocessing image algorithms to develop other industrial applications like identification, sorting, pattern analysis to detect the defective object, quality control, and assembly. With the single vision system, the algorithm controlled the conveyor at any time instant to perform operations. This method also has an advantage, that is, manipulating the objects both in a moving conveyor or intermittent motion conveyor. In intermittent conveyor, the objects are picked in a particular position, and the objects are separated based on the extracted features from the GMM approach. In the moving conveyor, the moving objects' center coordinates are extracted with time using GMM background subtraction. The synchronized center coordinates of the moving objects are mapped with a robot base coordinate system to effectively tackle the object. Based on the conveyor's speed, the robot actuates the end effectors for manipulation of the objects. This approach has autonomous controlling of the robot by developing the self-sustain routine to reach the target object. With the use of a single camera, the approach is limited to extract the object having same height because of the 2D image. The results indicated that the GMM background subtraction is superior when compared to temporal difference and direct subtraction background subtraction method. The relative advantages of GMM are indicated in Table 7.

Table 7. Performance Comparison of various algorithms in extracting the features.

\begin{tabular}{|c|c|c|c|}
\hline $\begin{array}{c}\text { Background subtraction } \\
\text { Method }\end{array}$ & Color retention & Illumination & $\begin{array}{c}\text { Detection Object with the } \\
\text { same background }\end{array}$ \\
\hline Temporal difference & Original & Constant & Not possible \\
\hline Direct subtraction & Pseudocolor & - & Not possible \\
\hline GMM & Original & Variable & Possible \\
\hline
\end{tabular}

\section{CONCLUSION}

The vision algorithm provides accurately reliable and real-time tracking information to activate the robot with conjunction moving object's position. In this approach, the robot moves to pick the object based on angle computed using its location information extracted from the image. The prototype successfully evaluated the accuracy of the integrated tacking algorithm. The algorithm reduces $3 \mathrm{D}$ work volume to a $2 \mathrm{D}$ plane extended to compute the joint angles to reach the target position, and by using planer inverse kinematics, the object is picked. The developed methodology is not suitable to pick the object having different heights, but it is suitable to pick the objects having different other features like shape, color, and patterns.

\section{ACKNOWLEDGMENT}

AICTE New Delhi funded this project under Research Promotion Scheme. 


\section{REFERENCES}

S Cheung Sen-Ching and Chandrika Kamath.2004 Robust techniques for background subtraction in urban traffic video. In Visual Communications and Image Processing 2004, volume 5308, pages 881-893.

Abbood, W.T., Abdullah, O.I. \& Khalid, E.A. (2020) A real-time automated sorting of robotic vision system based on the interactive design approach. Int $J$ Interact Des Manuf 14, 201-209. https://doi.org/10.1007/s12008-019-00628-w

Thotapalli, P.K., Vikram Kumar, C.R. \& Chandra Mohana Reddy, B. (2020) Feature extraction of moving objects using background subtraction technique for robotic applications. Int J Intell Robot Appl . https://doi.org/10.1007/s41315-020-00145-0

Chen L., Yang H., Liu P. (2019) Intelligent Robot Arm: Vision-Based Dynamic Measurement System for Industrial Applications. In: Yu H., Liu J., Liu L., Ju Z., Liu Y., Zhou D. (eds) Intelligent Robotics and Applications. ICIRA 2019. Lecture Notes in Computer Science, vol 11744. Springer, Cham. https://doi.org/10.1007/978-3-030-27541-9_11

Christof Ridder, Olaf Munkelt, and Harald Kirchner. 1995 Adaptive background estimation and foreground detection using Kalman-filtering. In Proceedings ofInternational Conference on Recent Advances in Mechatronics, pages 193-199. Citeseer,.

Chris Stauffer and W Eric L Grimson.1999 Adaptive background mixture models for real-time tracking.In Proceedings. 1999 IEEE Computer Society Conference on Computer Vision and Pattern Recognition (Cat. No PR00149), volume 2, pages 246-252. IEEE,

P Wayne Power and Johann A Schoonees 2002. Understanding background mixture models for foreground segmentation. In Proceedings image and vision computing New Zealand.

Massimo Piccardi.2004 Background subtraction techniques: a review. In 2004 IEEE International Conference on Systems, Man and Cybernetics (IEEE Cat. No. 04CH37583), volume 4, pages 3099-3104.

Roy De Maesschalck, Delphine Jouan-Rimbaud, and Désiré L Massart. 2000 The Mahalanobis distance. Chemometrics and intelligent laboratory systems, 50(1):1- 18

Alan M McIvor.2000 Background subtraction techniques. Proc. of Image and Vision Computing, 4:3099-3104.

P. St-Charles, G. Bilodeau and R. Bergevin,2015"SuBSENSE: A Universal Change Detection Method With Local Adaptive Sensitivity," in IEEE Transactions on Image Processing, vol. 24, no. 1, pp. 359-373.

K. Goyal and J. Singhai,2017 "Review of background subtraction methods using gaussian mixture model for video surveillance systems," Artificial Intelligence Review, pp. 1-19,

Thierry Bouwmans, Fida EI Baf, Bertrand Vachon.2008 Background Modeling using Mixture of Gaussians for Foreground Detection - A Survey. Recent Patents on Computer Science, Bentham Science Publishers 1 (3), pp.219-237

Thotapalli P.K., Vikram Kumar C.R., Chandra Mohana Reddy B. (2020) A New Approach to Control the Position of Joint Arm Robot Using Image Background Subtraction Technique. In: Shunmugam M., Kanthababu M. (eds) Advances in Simulation, Product Design and Development. Lecture Notes on Multidisciplinary Industrial Engineering. Springer, Singapore https://doi.org/10.1007/978-981-32-94875_71

Andrews Sobral and Antoine Vacavant(2014) A comprehensive review of background subtraction algorithms evaluated with synthetic and real videos. Computer Vision and Image Understanding, 122:4-21, 